\title{
Enantioselective $\beta$-Lactone Formation from Ketene and Aldehydes Catalyzed by a Chiral Oxazaborolidine
}

\author{
Vijay Gnanadesikan, E. J. Corey* \\ Department of Chemistry and Chemical Biology \\ Harvard University, 12 Oxford Street, Cambridge, Massachusetts 02138
}

$\underline{\text { Supplementary Materials }}$

Materials and Methods. Unless stated otherwise, reactions were performed in flame-dried glassware under a positive pressure of nitrogen using freshly distilled dry solvents. Thin-layer chromatography (TLC) was performed using E. Merck silica gel $60 \mathrm{~F}_{254}$ precoated plates $(0.25$ $\mathrm{mm})$. Flash chromatography was performed using Baker silica gel (40 $\mu \mathrm{m}$ particle size). NMR spectra were recorded on Varian Innova-500, or Mercury-400 instruments and calibrated using residual undeuterated solvent as an internal reference. IR spectra were recorded on Avatar 360 FT-IR spectrometer. Low-resolution and high-resolution mass spectral analyses were performed at the Harvard University Mass Spectrometry Center. Analytical high performance liquid chromatography (HPLC) was performed on Isco 2350 Series or Waters 626 HPLC using the indicated chiral column. Gas chromatography (GC) analyses were performed on HewlettPackard 6850 Series GC System equipped with flame ionization detector using indicated column (30 m $\times 0.25 \mathrm{~mm})$. Commercial grade reagents and solvents were used without further purification except as indicated below. Dichloromethane and $\mathrm{MeCN}$ were distilled from calcium hydride. 


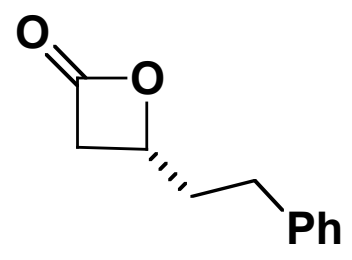

(R)-4-(2-Phenylethyl)oxetane-2-one. ${ }^{1}$ The general procedure was followed employing $66 \mu \mathrm{l}$ of 3-phenylpropanol $(0.5 \mathrm{mmol})$. Purification by flash chromatography gave $64 \mathrm{mg}(73 \%)$ of $\beta$ lactone. Analysis of enantiomers by chiral HPLC (Daicel Chiralcel OD-H column, flow rate 1 $\mathrm{mL} / \mathrm{min}, 10 \%$ IPA, 90\% hexane, $\mathrm{T}_{\mathrm{r}} 15.9(\mathrm{~S})$ and 18.1(R) provided the enantiomeric ratio: $4(\mathrm{~S}): 4(\mathrm{R})=90: 9(81 \% \mathrm{ee}) .[\alpha]_{\mathrm{D}}^{25}+5.62\left(c 0.32, \mathrm{CHCl}_{3}\right)$. Spectral data has been reported previously. ${ }^{1}$ IR (film) 3029, 2933, 1821, 1740, 1455, 1412, 1133, $1113 \mathrm{~cm}^{-1}$; ${ }^{1} \mathrm{H}$ NMR (600 MHz, $\left.\mathrm{CDCl}_{3}\right) \delta$ 2.06-2.12 (m, 1H), 2.18-2.24 (m, 1H), 2.72-2.77 (m, 1H), 2.82-2.87 (m, 1H), $3.05(\mathrm{dd}$, $J=4.2,18 \mathrm{~Hz}, 1 \mathrm{H}), 3.49(\mathrm{dd}, J=5.4,16 \mathrm{~Hz}, 1 \mathrm{H}), 4.50-4.53(\mathrm{~m}, 1 \mathrm{H}), 7.22-7.35(\mathrm{~m}, 5 \mathrm{H})$.

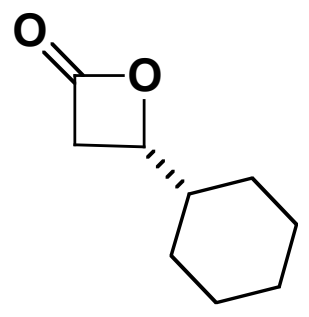

(S)-4-Cyclohexyloxetane-2-one. ${ }^{1}$ The general procedure was followed employing $60.5 \mu \mathrm{l}$ of cyclohexane carboxaldehyde $(0.5 \mathrm{mmol})$. Purification by flash chromatography gave $60 \mathrm{mg}$ (78\%) of $\beta$-lactone. Analysis of the enantiomers by chiral GC (cyclosil B column, flow rate $1 \mathrm{ml} / \mathrm{min}$ at $110^{\circ} \mathrm{C}, \mathrm{T}_{\mathrm{r}} 31.3$ (major) and 32.7 (minor) min) provided the enantiomeric ratio: $4(\mathrm{~S}): 4(\mathrm{R})=85: 15(70 \%$ ee $) . \quad[\alpha]_{\mathrm{D}}^{25}+2.12\left(c 0.33, \mathrm{CHCl}_{3}, 70 \%\right.$ ee $) . \quad$ Spectral data has been reported previously. ${ }^{1}$ IR (film) 2968, 2881, 1818, 1760, 1470, $1125 \mathrm{~cm}^{-1} ;{ }^{1} \mathrm{H}$ NMR (600 MHz, $\left.\mathrm{CDCl}_{3}\right) \delta$ 0.95-1.06 (m, 2H), 1.10-1.34 (m, 4H), 1.57-1.87 (m, 4H), 1.93-1.94 (m, 1H), $3.10(\mathrm{dd}$, $J=4.8,12 \mathrm{~Hz}, 1 \mathrm{H}), 3.41(\mathrm{dd}, J=6,16.8 \mathrm{~Hz}, 1 \mathrm{H}), 4.17-4.19(\mathrm{~m}, 1 \mathrm{H})$. 


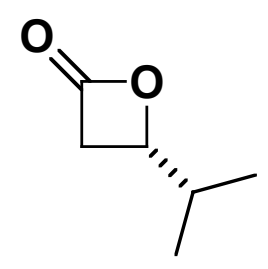

(S)-4-Isopropyloxetane-2-one. ${ }^{1}$ The general procedure was followed employing $45.5 \mu \mathrm{l}$ of isobutyraldehyde $(0.5 \mathrm{mmol})$. Purification by flash chromatography gave $35.5 \mathrm{mg}(62 \%)$ of $\beta$ lactone. Analysis of the enantiomers by chiral GC (Chiraldex $\gamma$-TA column, flow rate $0.5 \mathrm{ml} / \mathrm{min}$ at $100^{\circ} \mathrm{C}, \mathrm{T}_{\mathrm{r}} 27.4$ (major) and 30.5 (minor) $\mathrm{min}$ ) provided the enantiomeric ratio: $4(\mathrm{~S}): 4(\mathrm{R})=$ 84:16 (68\% ee). $[\alpha]_{\mathrm{D}}^{25}+4.00\left(c 0.25, \mathrm{CHCl}_{3}, 68 \%\right.$ ee). Spectral data has been reported previously. ${ }^{1}$ IR (film) 2931, 2838, 1821, 1711, 1451, 1277, $1129 \mathrm{~cm}^{-1} ;{ }^{1} \mathrm{H}$ NMR (600 MHz, $\left.\mathrm{CDCl}_{3}\right) \delta 0.94(\mathrm{~d}, J=6.6 \mathrm{~Hz}, 3 \mathrm{H}), 1.03(\mathrm{~d}, J=6.6 \mathrm{~Hz}, 3 \mathrm{H}), 1.91-1.95(\mathrm{~m}, 1 \mathrm{H}), 3.08(\mathrm{dd}, J=4.2$, 14.4 Hz, 1H), $3.42(\mathrm{dd}, J=6,22.2 \mathrm{~Hz}, 1 \mathrm{H}), 4.17-4.20(\mathrm{~m}, 1 \mathrm{H})$.

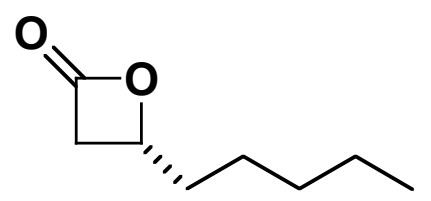

(S)-4-Pentyloxetane-2-one. ${ }^{2}$ The general procedure was followed employing $60 \mu \mathrm{l}$ of hexanol (0.5 mmol). Purification by flash chromatography gave $55.5 \mathrm{mg}(78 \%)$ of $\beta$-lactone. Analysis of the enantiomers by chiral GC (Chiraldex $\gamma$-TA column, flow rate $0.5 \mathrm{ml} / \mathrm{min}$ at $100^{\circ} \mathrm{C}, \mathrm{T}_{\mathrm{r}} 41.9$ (major) and $44.2\left(\right.$ minor) min) provided the enantiomeric ratio: $4(\mathrm{~S}): 4(\mathrm{R})=92: 8(84 \% \mathrm{ee}) .[\alpha]_{\mathrm{D}}{ }^{25}$ +3.04 (c 0.23, $\mathrm{CHCl}_{3}, 84 \%$ ee). Spectral data has been reported previously. ${ }^{2}$ IR (film) 2964, 2935, 1864, 1821, 1708, 1239, 1129, $1011 \mathrm{~cm}^{-1} ;{ }^{1} \mathrm{H}$ NMR $\left(600 \mathrm{MHz}, \mathrm{CDCl}_{3}\right) \delta$ 0.82-0.85 (m, $3 \mathrm{H}), 1.12-1.39(\mathrm{~m}, 6 \mathrm{H}), 1.66-1.71(\mathrm{~m}, 1 \mathrm{H}), 1.76-1.80(\mathrm{~m}, 1 \mathrm{H}), 2.99(\mathrm{dd}, J=5.4,19.8 \mathrm{~Hz}, 1 \mathrm{H})$, $3.45(\mathrm{dd}, J=6.6,19.2 \mathrm{~Hz}, 1 \mathrm{H}), 4.43-4.46(\mathrm{~m}, 1 \mathrm{H})$. 


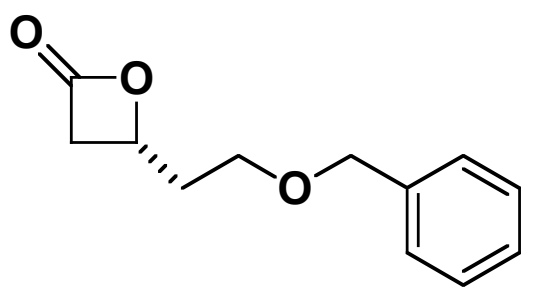

(R)- 4-(2-Benzyloxyethyl)-oxetane-2-one. ${ }^{1}$ The general procedure was followed employing 84 $\mathrm{mg}$ of 3-benzyloxypropionaldehyde $(0.5 \mathrm{mmol})$. Purification by flash chromatography gave 60 $\mathrm{mg}(58 \%)$ of $\beta$-lactone. Analysis of the enantiomers by chiral HPLC (Chiralcel OD-H column, flow rate $0.9 \mathrm{ml} / \mathrm{min} 15 \% \mathrm{IPA}, 85 \%$ hexane, $\mathrm{T}_{\mathrm{r}} 13.5$ (minor) and 17.0 (major) min) provided the enantiomeric ratio: $4(\mathrm{~S}): 4(\mathrm{R})=17: 82(65 \%$ ee $) .[\alpha]_{\mathrm{D}}^{25}+9.75\left(c 0.80, \mathrm{CHCl}_{3}, 65 \%\right.$ ee $)$. Spectral data has been reported previously. ${ }^{1}$ IR (film) 2925, 2860, 1823, 1725, 1455, 1362, 1117, 1102 $\mathrm{cm}^{-1} ;{ }^{1} \mathrm{H}$ NMR (600 MHz, $\mathrm{CDCl}_{3}$ ) $\delta$ 2.11-2.17 (m, 2H), $3.24(\mathrm{dd}, J=4.2,16.8 \mathrm{~Hz}, 1 \mathrm{H}), 3.57$ (dd, $J=5.4,16.2 \mathrm{~Hz}, 1 \mathrm{H}), 3.5-3.68(\mathrm{~m}, 2 \mathrm{H}), 4.54(\mathrm{~s}, 2 \mathrm{H}), 4.74-4.76(\mathrm{~m}, 1 \mathrm{H})$.

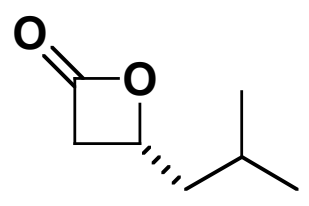

(R)- 4-(2-Methylpropyl)-oxetane-2-one. ${ }^{1}$ The general procedure was followed employing 53.6 $\mu 1$ of isovalaraldehyde $(0.5 \mathrm{mmol})$. Purification by flash chromatography gave $46.3 \mathrm{mg}(72 \%)$ of the $\beta$-lactone. Enantiomers were analyzed, after lactone opening with benzylamine to form the benzylamide, by chiral HPLC (Chiralcel OD-H column, flow rate 1ml/min 5\% IPA, 95\% hexane, $\mathrm{T}_{\mathrm{r}} 8.4$ (major) and 13.4 (minor) min) provided the enantiomeric ratio: $4(\mathrm{~S}): 4(\mathrm{R})=83: 16(67 \%$ ee). $[\alpha]_{\mathrm{D}}{ }^{25}+7.33\left(c 0.15, \mathrm{CHCl}_{3}, 67 \%\right.$ ee). Spectral data has been reported previously. ${ }^{1}$ IR (film) 2964, 1864, 1823, 1710, 1372, 11239, $1113 \mathrm{~cm}^{-1} ;{ }^{1} \mathrm{H} \mathrm{NMR}\left(600 \mathrm{MHz}, \mathrm{CDCl}_{3}\right) \delta 0.94(\mathrm{~d}, J$ 
$=6 \mathrm{~Hz}, 6 \mathrm{H}), 1.56-1.6(\mathrm{~m}, 1 \mathrm{H}), 1.77-1.81(\mathrm{~m}, 2 \mathrm{H}), 3.02(\mathrm{dd}, J=4.2,10.2 \mathrm{~Hz}, 1 \mathrm{H}), 3.52(\mathrm{dd}, J=6$, 16.8 Hz, 1H), 4.54-4.57 (m, 1H).

\section{References.}

1) Nelson, S. G.; Peelen, T. J.; Wan, Z. J. Amer. Chem. Soc. 1999, 121, 9742-9743.

2) Schmidt, J. A. R.; Lobkovsky, E. B.; Coates, G. W. J. Amer. Chem. Soc. 2005, 127, 11426-11435. 